\title{
Study of Plantar Pressure Distribution
}

\author{
Chun Kit Ang ${ }^{1, a}$, Mahmud Iwan Solihin ${ }^{1}$, Weng Jun Chan ${ }^{1}$ and Yien Yien Ong ${ }^{2}$. \\ ${ }^{1}$ Department of Mechanical and Mechatronic Engineering, UCSI University, No.1, Jalan Menara Gading, UCSI \\ Heights (Taman Connaught), 56000 Cheras, Kuala Lumpur, Malaysia. \\ ${ }^{2}$ Department of Bioscience, Faculty of Applied Sciences (FOAS), Tunku Abdul Rahman University College (TARUC), \\ Jalan Genting Kelang, Setapak, 53300 Kuala Lumpur, Malaysia.
}

\begin{abstract}
Every region of foot is not equally divided in terms of plantar pressure distribution (PPD) during free standing. This paper is focusing on studying PPD on flat plane and inclined plane and the results obtained from this study may contribute to biomedical researcher in designing orthotic devices. 24 healthy young adults age ranging from 19 to 24 years old and weigh between 50 to $80 \mathrm{~kg}$ were invited for experiments purpose. Six regions of both feet were measured which were hallux, medial forefoot, central forefoot, lateral forefoot, lateral midfoot and hindfoot. Remarkable differences were seen in the result as right foot exerted more pressure generally in every region of the foot as to compared with left foot respectively. This is true especially for region such as hallux, medial forefoot and lateral forefoot. On a flat surface, PPD on the hindfoot is the highest. However, at an elevation of $25^{\circ}$, test subjects began to shift their PPD to forefoot regions. While studies of PPD are common, this study provides a new insight for the first time into PPD while standing on different angle of walking plane.
\end{abstract}

\section{Introduction}

Most individual spend a large proportion of their lifetimes in the standing position. Walking also known as ambulation is one of the fundamental locomotion for a human being and is the most common activity in our daily life. During standing, our feet and the surface of the ground created a contact. The sole of our feet experienced a force upon landing on the ground. The force of impact on the sole of the foot or the plantar pressure distribution could be irregular and unbalanced. These impacts manipulate changes in pattern of stress applied to the foot, stresses that may causes temporary or permanent strains on the tissues of the foot and therefore induces foot conditions. In a long run, this may cause a foot injury or worsen the disruption of overall health of an individual [1].

Human foot plantar pressure refers to pressure acting upon on the soles of human feet when stepping on surface of ground. In today eras, plantar pressure distributions are profoundly accepted as a reliable biomechanical parameter to study and diagnose various foot disorders. Plantar pressure distributions are affected by various factors such as weight, gender, foot structure and even the way an individual stands or walks. Plantar pressure measurements during standing, walking or other activities provides detailed information for evaluation of diseases or abnormalities, involving the function of the ankle, knee, hip, the back and other patho-mechanics and yield objectives measure to track disease

${ }^{a}$ Corresponding author: ack_kit@hotmail.com 
progression [2]. It also provides vital information on mechanical behaviour of the human foot in both static and dynamic conditions.

In the past, a number of studies on foot plantar pressure have been carried out. The study of relationship between plantar foot pressure and supporting surface is called pedobarography. It is often studied for bio-mechanical analysis of human walking or gait analysis and human posture. Pedobarography is extensively practiced and clinically applied in patients with diabetic foot ulceration, a condition which may lead to amputation in extreme cases. Several research groups acknowledge the potential of plantar pressure distributions technology for the diagnosis and analysis of different foot abnormality. In fact, few studies on foot biomechanics have found that plantar pressure distributions are vital to determine the unnatural gait [2].

In most studies, plantar pressure distributions are usually examined during walking. However, studies on plantar pressure distributions while standing on different inclined plane are rare. The knowledge of the changes in plantar pressure distribution of a person standing on an inclined platform may be helpful to further understand the neuropathological mechanism to make physical activities safe and to prevent injury.

\section{Experiment Setup}

\subsection{Sensor}

Traditionally, stationary force platforms are used to measure the foot plantar pressure distributions as shown in Figure 1. They are efficient when comes to measuring force applied directly on the platform surfaces. But over the years, a new trend of insole techniques is growing rapidly. The sensor used are typically more flexible and can be placed in flat or curves surfaces. These systems detect the plantar pressure and the shoe that were put on directly and therefore can be custom made accordingly in terms of shoe construction. A general advantage of in-sole technique is that data of multiple steps can be recorded in a single test [3]. Since the position of the foot is mounted on the respective sensor, the data of multiple steps can be averaged easily, thus reducing measurement error, in contrast with platform devices which has only one ground contact that can be generated at a time. Portability plays an important role in collection of data. It encourages a more natural gait movement among test subjects whereas for flat platform, test subjects have to anticipate each and every step so that they can accurately step on the platform itself.

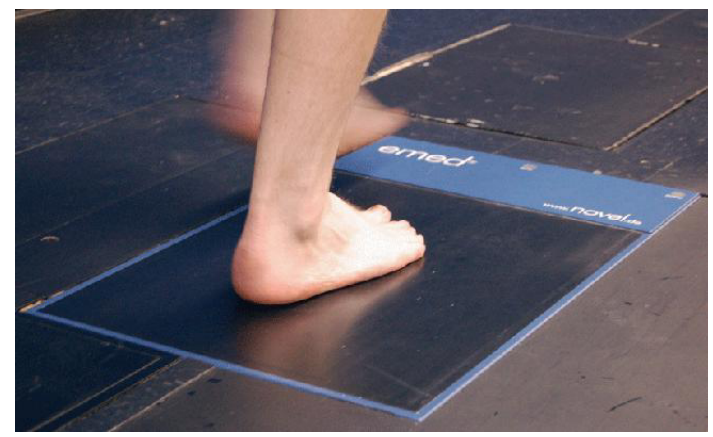

Figure 1. A Platform-Based Foot Plantar Pressure Sensor [4]

Thus, the in-sole sensors that are used in this study are the force sensing resistors (FSRs). FSR is a robust polymer thick film (PFT) components that will decrease in resistance as force applied to the sensing area. The model of the transducer that will be used in this study is the IMS-C20B. The reason this model is chosen compared to its counterpart is because this model may withstand and measure higher force up to $100 \mathrm{~kg}$ which facilitate in this study. It has a small sensing area of about $20 \mathrm{~mm}$ in diameter. This allow accurate readings to be taken. Apart from that FSR is generally more robust than 
any other sensor due to its nature to withstand strains and stresses. It is made from ultra-thin filaments, about $0.45 \mathrm{~mm}$ thus it is easy to be placed under the sole of the foot and minimizes the error due to its sizes. Besides, FSR has a high repeatable force reading which plays an important role in data collection for this measuring device as test subjects are required to walk on the sensor in repeatable manner. All these factors are to be considered as they can potentially increase the measurement error and device failure.

\subsection{Foot region}

There have been many studies conducted that are based on the foot of a person. However, PPD is not equally distributed across every region of the foot. In many studies, researchers have their own set of regions of foot of interest. For example, according to Gurney, Kersting and Rosenbaum (2008) the foot was divided into multiple regions which are: Hallux (HA); Second toe (T2); Lateral toes (T3-5); Lateral forefoot (LF); Central forefoot (CF); Medial forefoot (MF); Lateral midfoot (LM); Medial midfoot (MM); Lateral hindfoot (LH); Medial hindfoot (MH) [5] as shown in Figure 2.

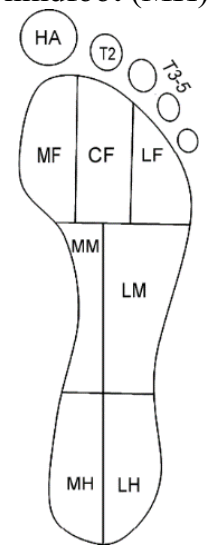

Figure 2. Division of Foot Anatomical Areas of Interest [5]

Similarly, in this study, each foot is divided into 6 regions to enable analysis of every significant region in relation to pressure. The placement of sensors is shown in Figure 3. Region of foot are as following: Hallux (HA); Medial Forefoot (MF); Central Forefoot (CF); Lateral Forefoot (LF); Lateral Midfoot (LM); Hindfoot (HF).

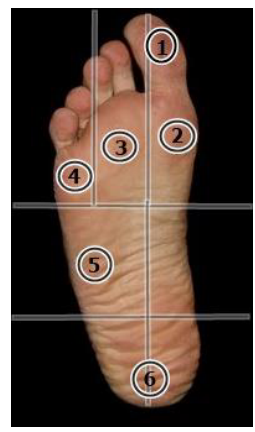

Figure 3. Division of Region of Foot and the Placement of Sensor

\subsection{Test subjects}

The test subjects in this study consisted of 24 healthy volunteers without any foot disorder, including 14 males and 10 females age ranging from 19 to 24 years old and weighed between 50 to $80 \mathrm{~kg}$. Age, 
weight and gender were recorded for each test subject to provide information of the sample data taken. Prior to the experiment, test subjects were briefed about the experimental procedure and given some time to familiarize themselves with the procedure. The subjects were instructed to stand on the platform (as shown in Figure 4) at their natural standing position. Test subjects underwent six different tests as there were six inclined planes. Five readings were taken for every type of test to minimizes error.

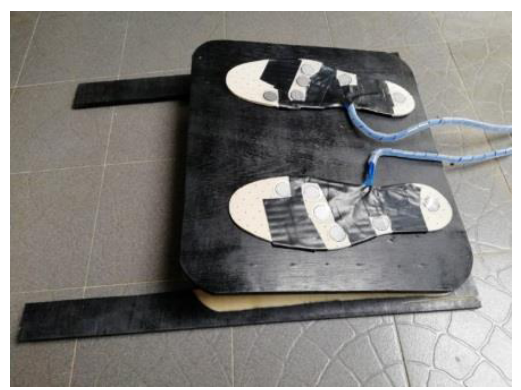

Figure 4. Adjustable Inclined Platform

\subsection{Testing plane}

As mentioned earlier, there were six inclined planes with different elevation were tested in this study. The inclined plane was hard and sturdy surfaces to simulate the hard ground upon contact of foot. As shown in Figure 4, these were the following inclined planes: flat plane; $5^{\circ}$ inclined plane; $10^{\circ}$ inclined plane; $15^{\circ}$ inclined plane; $20^{\circ}$ inclined plane; $25^{\circ}$ inclined plane.

\section{Results and Discussion}

In this study, PPD for both feet are measured and recorded simultaneously. There are few factors that affect the value such as gender, age, and anatomical structure of the foot. Therefore, there are many ways to analyze the result obtained. The loading of human foot on the ground surfaces is not constant. In Figure 5, the results show an ever changing of pressure on each region of foot for one of the test subjects. The changes are not consistent and unpredictable. It may change to an extend range of 55\% of the pressure in a span of 10 seconds. This was observed on every test subjects despite being instructed to stand still. The result is agreeable to the studies conducted by Kei Masani et. al in her work [6]. All the other test subjects have the similar results. In order to reduce the inaccuracy, the test subject was instructed to stand still for 10 seconds and the average value of the readings for each region of the foots was recorded.

The pie chart in Figure 6 shows the average distribution of pressure of both feet of all the test subjects on flat plane. According to the chart, hindfoot exerts the most pressure which is nearly one third of the entire foot pressure combined. The second highest plantar pressure exerted is the medial forefoot which is $19 \%$, followed by central forefoot, $18 \%$. Lateral forefoot exerts $17 \%$ of the plantar pressure and Hallux exerts 10\%. Lastly, Lateral Midfoot exerts the least pressure of the foot which is $4 \%$. The order from highest PPD to the lowest is as followed: $\mathrm{HF}>\mathrm{MF}>\mathrm{CF}>\mathrm{LF}>\mathrm{HA}>\mathrm{LM}$.

This validates a previous study [7] in adult by Klimiec in 2016 which found the highest PPD to be at hindfoot region which follow by forefoot region and hallux . Lastly, midfoot region exerted the least pressure among all.

Besides, this study discovers that the loading pressure of the right foot varies with the left foot. Remarkable differences were seen in the result as right foot exerted more pressure generally in every region of the foot as to compare with left foot respectively as shown in Figure 7. This is true especially for region such as hallux, medial forefoot and lateral forefoot. 


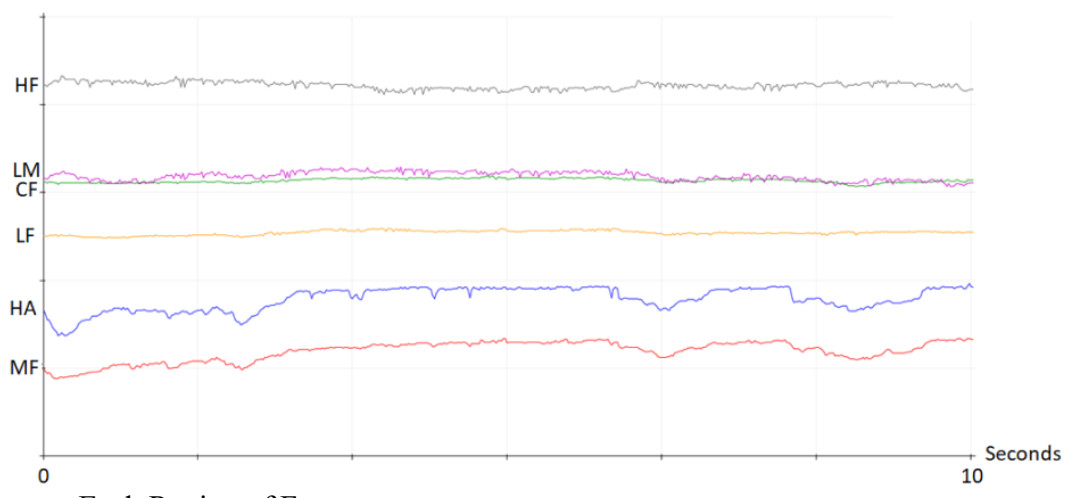

Figure 5. Force on Each Region of Foot

\section{Plantar Pressure Distribution}

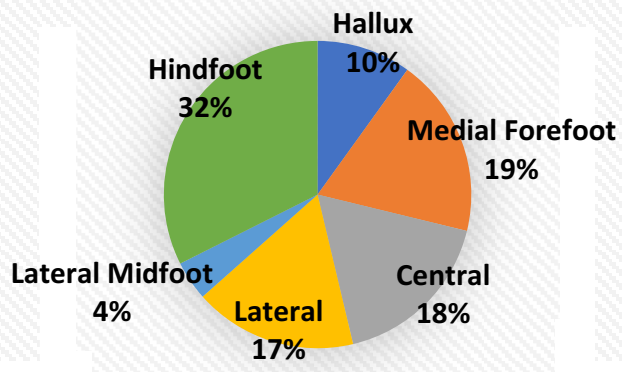

Hallux $\square$ Medial Forefoot Central L Lateral $\square$ Lateral Midfoot $\square$ Hindfoot

Figure 6. Pie Chart of Plantar Pressure Distribution

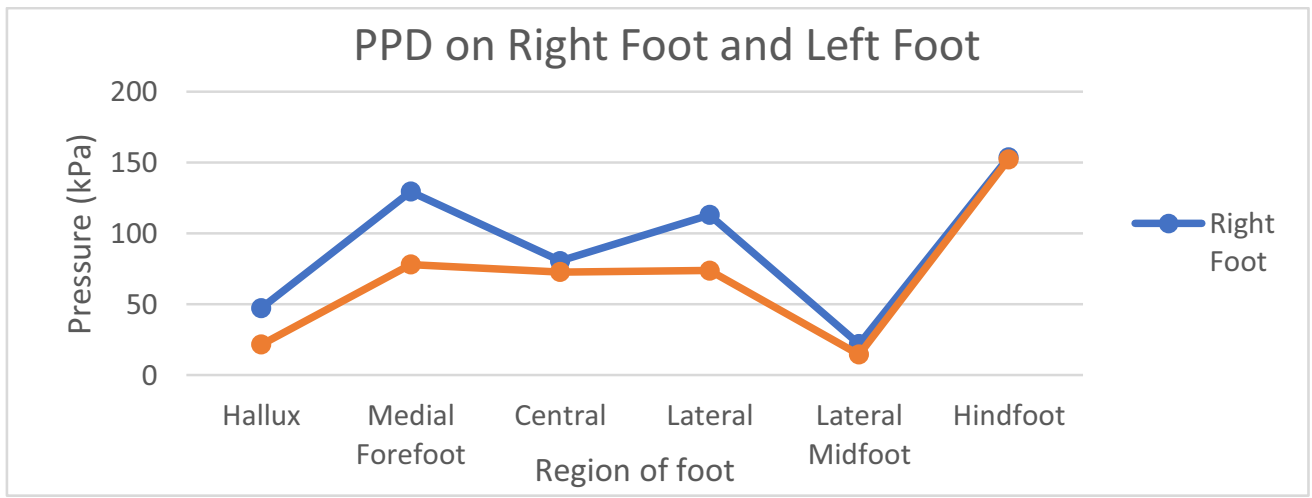

Figure 7. Comparison of Plantar Pressure on Different Regions of Feet

Figure 8 to Figure 11 show the differences of PPD for male and female. Previous studies by Periyasamy in 2011 reported that no significant differences was detected when comparing PPD of men and women of both foot on flat surfaces and mentioned that there were no differences among genders in the midfoot contact area [8]. Similarly, on inclined plane, there are no significant PPD differences between male and female. However, this study discovers that the main difference is the PPD on hindfoot region. The PPD on hindfoot of female is slightly higher than male while standing on inclined plane. This may due to higher contact area of men compared to women. 


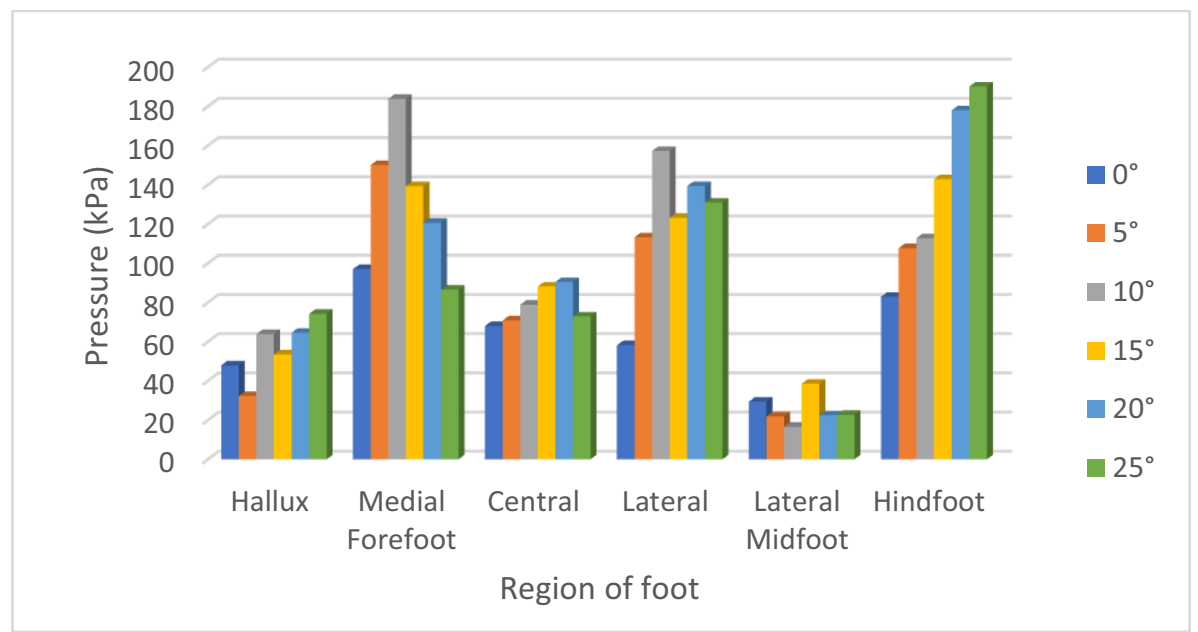

Figure 8. Plantar Pressure of Male Subjects on Right Foot

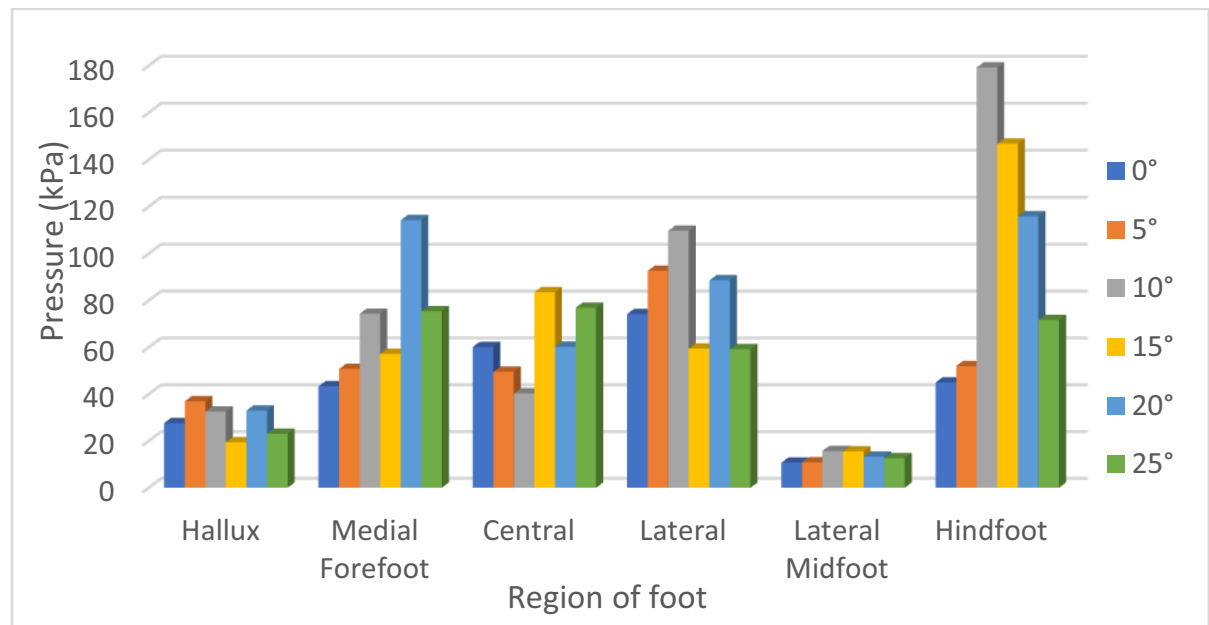

Figure 9. Plantar Pressure of Male Subjects on Left Foot

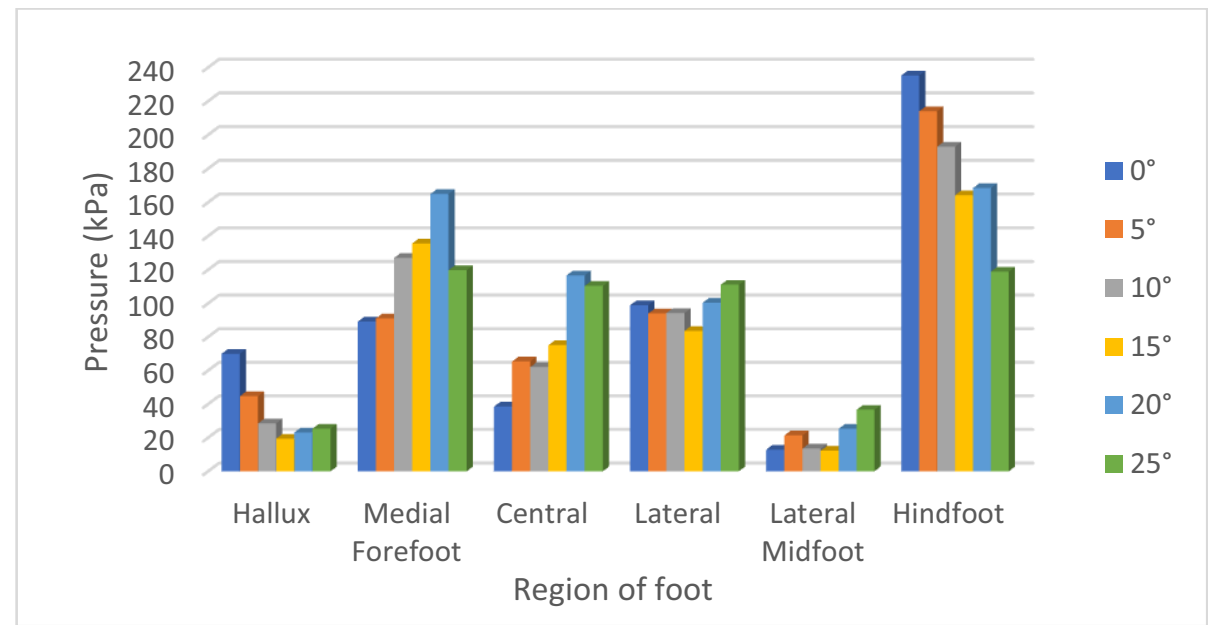

Figure 10. Plantar Pressure of Female Subjects on Right Foot 


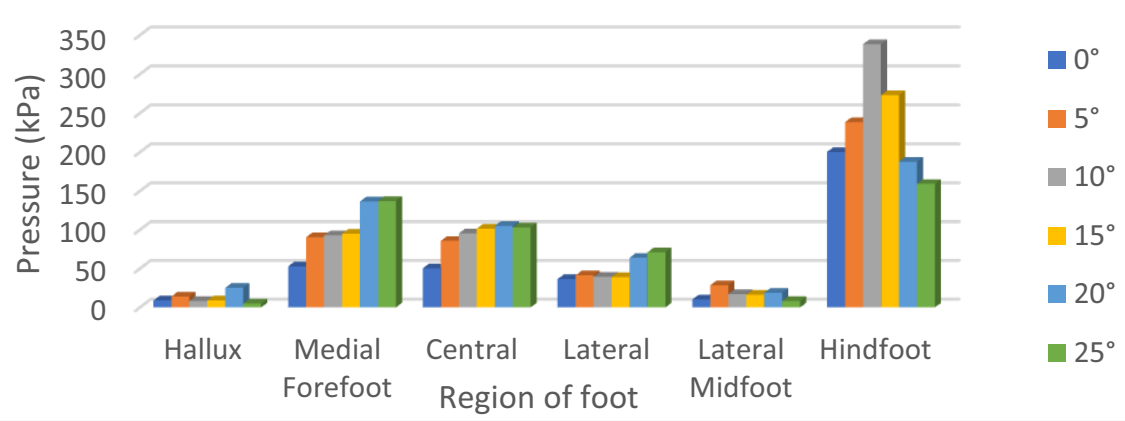

Figure 11. Plantar Pressure of Female Subjects on Left Foot

Furthermore, an astonishing result were shown in Figure 12 as the elevation changes, human feet shifted to compensate the body instability. The shift mainly focused on the medial forefoot, central forefoot, lateral forefoot and the hindfoot. On a flat surface, PPD on the hindfoot is the highest. However, at an elevation of $25^{\circ}$, test subjects began to shift their PPD to the forefoot regions. This is due to human body leaning slightly forward thus changes in pressure distribution to avoid falling backwards. At $30^{\circ}$ slope, it is observed that many test subjects failed to balance themselves on the platform. Therefore, the $30^{\circ}$ slope test were excluded for this study.

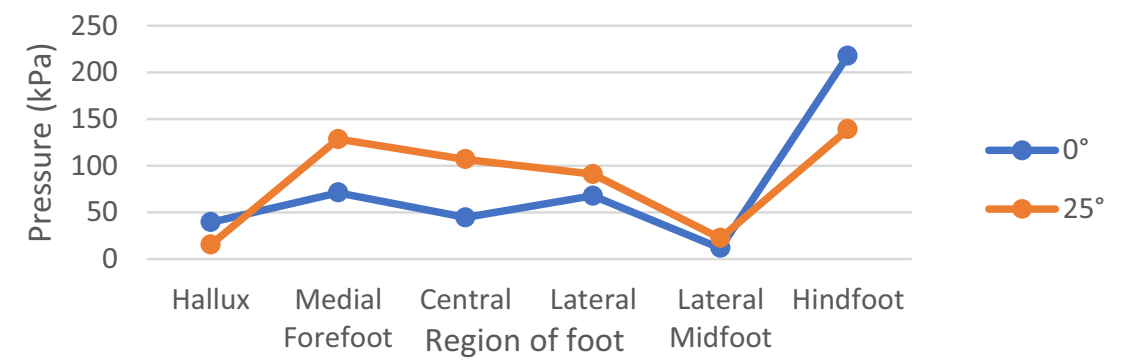

Figure 12. Comparison of $0^{\circ}$ and $25^{\circ}$ on Both Feet

\section{Conclusion}

Plantar pressure distribution measurement is extremely useful in analyzing the weight loading of human foot. This study has evaluated that human feet are impossible to stand absolute still as muscles are continuously working to keep the person in balance. PPD on the hindfoot region is the highest while medial midfoot remains the lowest regardless of different inclined plane which less than $25^{\circ}$ and gender. Besides that, as the elevation increases, the human foot shifted its PPD to forefoot region to compensate the body instability. While studies of PPD is common, this study provides a new insight for the first time into PPD while standing on different inclined plane.

\section{References}

1. Y. T. Chen, K. W. L. and S.Y. Huang, Int J Min Metall Mech Eng (IJMMME) 3(2) 65-69 (2015).

2. J. A. R. Bautista, et al., Biocybern. Biomed. Eng. 38(2) 342-361 (2018).

3. D. Rosenbaum and H. P. Becker, Foot and Ankle Surg 3(1) 1-14 (1997).

4. A. H., Abdul Razak et al., Sensors (Switzerland), 12(7) 9884-9912 (2012).

5. J. K. Gurney, U. G. Kersting, and D. Rosenbaum, Gait and Posture 27(4) 706-709 (2008).

6. K. Masani, D. G. Sayenko, and A. H. Vette, Gait and Posture 37(1) 72-77 (2013).

7. E. Klimiec et al., Sens. Actuators, A 247 504-513 (2016).

8. R. Periyasamy et al., Foot 21(3)142-148 (2011). 